\title{
Una interpretación institucionalista de la política macroprudencial
}

\author{
Gustavo Chagas Goudard y Fábio Henrique Bittes Terra
}

Una de las consecuencias de la reciente crisis económica internacional fue la demanda de nuevos instrumentos de política económica, además de los ya consagrados mecanismos de política monetaria, cambiaria y fiscal. En particular, se procura buscar medios más eficaces para regular el sistema financiero e impedir el surgimiento de desequilibrios que afecten a la economía real. En ese sentido, la política macroprudencial se ha destacado como otra política pública de matriz económica cuya utilización puede contribuir al mantenimiento de la estabilidad financiera. No obstante, los debates y el desarrollo de la literatura sobre este tema se basan en fundamentos pragmáticos no relacionados directamente con las escuelas ortodoxas o heterodoxas de la ciencia económica. En ese contexto, el objetivo de este trabajo es realizar una lectura institucionalista de la política macroprudencial, sobre la base de que esta puede comprenderse con arreglo al contenido teórico de los enfoques institucionalistas.

PALABRAS CLAVE

CLASIFICACIÓN JEL

AUTORES
Crisis económica, política económica, política financiera, regulación económica, reglamento financiero, estabilización económica

$\mathrm{E} 60, \mathrm{E} 61, \mathrm{~B} 52$
Gustavo Chagas Goudard es Doctorando en Economía del Programa de Postgrado en Economía de la Universidad de São Paulo, Brasil. gustavogoudard@usp.br

Fábio Henrique Bittes Terra es Profesor del Instituto de Economía de la Universida Federal de Uberlândia, Brasil. fhbterra@ie.ufu.br 


\section{I}

\section{Introducción}

La reciente crisis económica puso en evidencia la necesidad de implementar un conjunto más amplio de políticas económicas, además de las ya consagradas intervenciones cambiaria, monetaria y fiscal. Los excesos que condujeron a la crisis crearon la demanda de nuevos mecanismos para limitar el alcance de la liberalización financiera, con miras a evitar las inflexiones cíclicas y sus graves consecuencias negativas en la producción, el empleo y la generación de riqueza. En tal sentido, la política macroprudencial ha cobrado importancia, tanto en la literatura académica sobre regímenes de política económica (Galati y Moessner, 2010; Landau, 2009), como en los discursos de los representantes de los organismos multilaterales, los bancos centrales y de los encargados de la formulación de políticas (Banco de Inglaterra, 2009; FMI, 2011; Blanchard, Dell'Ariccia y Mauro, 2010; y Bernanke, 2011).

Sin embargo, la política macroprudencial todavía es objeto de un debate inconcluso en la literatura económica, a tal punto que, por ejemplo, todavía no se ha definido si se debe hablar de política macroprudencial propiamente dicha o simplemente de medidas macroprudenciales ${ }^{1}$. No obstante, existe un cierto consenso en cuanto a los objetivos que orientan la actuación macroprudencial: esta apunta, sobre todo, a la supervisión y regulación de las instituciones financieras bancarias y no bancarias a objeto de contener el riesgo sistémico de insolvencia del sistema. La lógica consiste en mitigar las dos dimensiones del riesgo sistémico — carácter procíclico y entrelazamiento entre las instituciones nacionales y extranjeras- para lograr una estabilidad financiera más permanente. El desarrollo de la literatura sobre el tema se

\footnotetext{
${ }^{1}$ Desde el comienzo es necesario realizar una aclaración. Mientras en el Brasil se ha optado por hablar de medidas macroprudenciales, en la literatura extranjera se hace referencia a una política macroprudencial. Entonces, ¿es esta una política, entendida como régimen sistematizado de uso de los instrumentos macroprudenciales, coherente con las demás políticas económicas y llevada a cabo por una institución específica, o se trata simplemente de medidas dispersas y circunstanciales? En este trabajo se entiende que las políticas macroprudenciales pueden considerarse como una política, que incluye medidas de reglamentación del sistema financiero y es coherente con las demás políticas. De ese modo, las medidas macroprudenciales son las formas de aplicación de la política macroprudencial. En la literatura internacional, tanto institucional (FMI, 2011; Banco de Inglaterra, 2009, y BPI/FMI/CEF, 2011) como académica (Caruana, 2010; y Galati y Moessner, 2010), también se atribuye a las medidas macroprudenciales el estatus de política.
}

basa en fundamentos pragmáticos que no se relacionan directamente con las escuelas ortodoxas o heterodoxas de la ciencia económica, de modo que el debate sobre las políticas macroprudenciales se caracteriza por la ausencia de una perspectiva teórica que fundamente su utilización. Dicha ausencia justifica el empeño del presente trabajo.

En la teoría institucionalista se hace hincapié en las relaciones entre las esferas política y económica de la sociedad. Por ejemplo, Zysman (1983) argumenta que diferentes institucionalidades del sistema financiero, conformadas de manera activa por las políticas públicas, suponen formas divergentes de financiamiento y crecimiento de la economía. North (1990) pone de relieve la dinámica institucional de las sociedades como elemento central para el desarrollo económico que estas alcanzan. Para ello, algunos elementos de cuño político, como la garantía de los derechos de propiedad y el mantenimiento de la estabilidad de los contratos, asegurarían la reducción de los costos de transacción y el aumento de la eficiencia económica y conducirían a trayectorias de desarrollo favorables. A partir del concepto de nexo económico-legal (legal-economic nexus), Samuels (2007) resume el modo en que las políticas públicas se traducen en estrategias diversas de las empresas, dado que las reglamentaciones, definidas en el ámbito político de la sociedad, delimitan el perfil que tendrán los mercados. Tratando de conciliar las nociones institucionalistas y poskeynesiana, Conceição y Ferrari-Filho (2001) argumentan que la idea keynesiana de socialización de las inversiones puede entenderse como la construcción, por medio de políticas públicas, de un ambiente institucional propicio a las inversiones productivas donde se mitiguen los movimientos cíclicos a que están sujetas las economías monetarias de producción. En consecuencia, no es una novedad que se utilice la teoría institucionalista, en sus diversas acepciones, para comprender la manera (cómo) en que las políticas públicas constituyen diferentes institucionalidades, que dan lugar a comportamientos individuales diversos y, en última instancia, a distintas trayectorias del sistema económico.

En ese contexto, el objetivo del presente trabajo consiste en realizar una lectura institucionalista de la política macroprudencial. El argumento en que se basa el artículo radica en que al analizar la referida política 
con arreglo al marco institucionalista, esta puede entenderse como creadora de una matriz institucional que conforma un sistema financiero más estable. Si bien tienen un carácter disperso — pues prevalece la ausencia de un régimen definido en términos de instrumentos, autoridades ejecutivas y metas intermediarias - se argumenta que las medidas macroprudenciales pueden funcionar como una política pública que restringe las operaciones financieras de mayor riesgo de las instituciones bancarias y no bancarias, y contribuye así a impedir las burbujas financieras y sus consiguientes efectos reales. Este artículo se inspira en un trabajo de Goudard y Terra (2013), en el que se realiza una lectura keynesiana de la política macroprudencial. En este caso se sigue una línea similar, pero con miras a contribuir al debate desde el punto de vista de la teoría institucionalista.
El artículo se divide en cuatro secciones, además de esta Introducción. En la segunda sección se presentan los enfoques del antiguo institucionalismo (también llamado viejo u original), el neoinstitucionalismo y la nueva economía institucional, y se aprovechan los aportes neoschumpeterianos que complementan la teoría institucionalista y contribuyen al análisis de las políticas macroprudenciales. A su vez, en la tercera sección se revisa la literatura sobre la política macroprudencial, incluidos sus orígenes, objetivos, instrumentos e interacciones con las demás políticas económicas. En la cuarta sección se establece una relación entre la segunda y la tercera secciones mediante el análisis de la política macroprudencial en un marco institucionalista. Por último, a modo de conclusión se presentan las consideraciones finales del trabajo.

\section{II}

\section{Los enfoques institucionalistas}

Samuels (1995) y Conceição (2002a) destacan que la riqueza del pensamiento institucionalista está en la pluralidad de enfoques que derivan de las diferentes escuelas que tratan el tema de las instituciones, aunque no siempre de forma convergente. En esas circunstancias, el propio concepto de institución adquiere diversos significados. Por una parte, el análisis del institucionalismo de acuerdo con un único enfoque empobrece su poder teórico y analítico, que tiene en la multiplicidad y la interacción sus expresiones más relevantes. Por otra parte, esa amplitud dificulta la exposición de todos los enfoques y plantea el riesgo de que las perspectivas sean incongruentes. Para resolver este dilema, sin perder de vista las virtudes del pluralismo, el método de presentación de este artículo consiste en trazar la historia de los enfoques, indicando el concepto de institución adoptado por cada uno de ellos. En particular, se describen las escuelas del antiguo institucionalismo, el neoinstitucionalismo y la nueva economía institucional.

De acuerdo con Hodgson (1993 y 1998); Conceição (2002a, 2002b y 2008); Samuels (1995), y Villeval (1995), entre otros, el pensamiento institucionalista deriva del viejo institucionalismo estadounidense de Thorstein Veblen, John Commons y Wesley Mitchell. Para esos autores, el concepto de institución se centra en el conjunto de hábitos, normas, valores, creencias, símbolos y patrones socialmente aprendidos y compartidos que suponen determinados comportamientos individuales y sociales, y su evolución. Para Veblen (1919, pág. 190), el institucionalismo es la "teoría del proceso de cambio consecutivo, considerado como una secuencia de cambio acumulativo, que se continúa y se propaga a sí mismo, y que no tiene término final", de modo que la situación de hoy moldea la institución de mañana por medio de un proceso selectivo y coercitivo, al actuar sobre la forma en que los hombres ven las cosas habitualmente. No es por menos que, para Commons (1931, pág. 648), las instituciones son acción colectiva en control, liberación y expansión de la acción individual. Hodgson (1993) caracteriza a los viejos institucionalistas diciendo que son en esencia evolucionistas y que ellos subrayan la importancia de comprender los procesos que alteran los elementos que constituyen las instituciones como medio para entender la dinámica del sistema económico. Se rechazan así las ideas neoclásicas de que el individuo es pasivo, independiente, inerte y con las preferencias dadas (conforme al individualismo metodológico) y de que las tecnologías de producción están dadas.

Dos conceptos formulados por los viejos institucionalistas son especialmente importantes para este trabajo: el de arraigo o integración (embeddedness) y lo que Veblen denomina "instituciones imbéciles" (imbecile institutions). El primero se refiere a la forma en que las instituciones se arraigan socialmente después 
de constituidas. Para Polanyi (1944), las instituciones surgen de manera no intencional (instituciones "orgánicas", que son reglas y costumbres que emanan de las interacciones individuales) o intencionalmente (instituciones "pragmáticas", establecidas para resolver un conflicto de interés individual, de grupo o social). Una vez percibida y comprendida, la institución pasa por un proceso de aceptación social que puede volverse efectiva o no, según la legitimidad y la valorización colectiva que reciba. Si la institución se incorpora al orden institucional y se comparte en el hábito, se dice que se ha arraigado o integrado.

El arraigo de instituciones que no construyen beneficios sociales configura lo que Veblen denominó “instituciones imbéciles" (Arthur, 1989), constituidas intencional o no intencionalmente, cuyos resultados de alguna forma causan dificultades en los sistemas en que están insertas. A modo de ejemplo, varias instituciones financieras (ya sean las propias empresas o los productos financieros) resultaron "imbéciles", pues a pesar de las ganancias momentáneas que permitieron, provocaron perjuicios que se extendieron mucho más allá del ámbito financiero de la economía ${ }^{2}$.

Después de algunas décadas de olvido, el institucionalismo fue rescatado en los años sesenta por los exponentes de dos enfoques: el neoinstitucionalismo y la nueva economía institucional. El primero constituye un rescate cabal del antiguo institucionalismo, incluso en la oposición al neoclasicismo, y las instituciones son tanto un hábito compartido, de acuerdo con Veblen (1919), como la acción colectiva, según Commons (1931). Hodgson (2002), uno de los precursores del neoinstitucionalismo, define las instituciones como sistemas duraderos de reglas sociales arraigadas y establecidas que estructuran las interacciones sociales ${ }^{3}$. Su durabilidad se debe a que las instituciones crean estabilidad en las expectativas y los hábitos individuales y permiten pensamientos y acciones ordenados porque dan forma y consistencia a las actividades humanas. De acuerdo con Hodgson (2004, pág. 652), para definir el término "instituciones" es fundamental tener en cuenta el concepto de hábito, que

\footnotetext{
${ }^{2}$ La crisis económica reciente constituye un ejemplo de los problemas que pueden derivarse de innovaciones financieras como la obligación de deuda garantizada, los papeles comerciales respaldados por activos y los bonos con opción de recompra, entre otros. Véanse ejemplos en Krugman (2009).

3 En esta acepción (que tiene un notable carácter histórico), el lenguaje, la moneda, el sistema financiero, el sistema de pesos y medidas y las empresas son algunos ejemplos de institución. Véase más en Hodgson (2002).
}

— junto con la propia institución— representa el núcleo duro del neoinstitucionalismo y debe entenderse como la propensión a comportarse de una determinada forma en una clase particular de situaciones.

Los individuos están influenciados por el ambiente institucional al que están sujetos (y en el que se encuentra la manifestación de la acción colectiva, ya sea intencional o no), que entrañará diferentes predisposiciones, es decir, hábitos diversos. Para Hodgson (2004), los hábitos no son los comportamientos reales de los individuos, sino la capacidad o propensión activa y reactiva que estos tienen para enfrentar la complejidad, la alternancia ambiental y la incertidumbre en las que están circunscritos en forma inherente. En consecuencia, si bien están estrechamente ligados, hábito y comportamiento no son la misma cosa. Si adquirimos un hábito, no necesariamente lo utilizamos todo el tiempo. Un hábito es una disposición a un comportamiento o pensamiento previamente adoptado o adquirido, provocado por un estímulo o contexto apropiados (Hodgson, 2006, pág. 6). Así, el hábito es una propensión a determinada forma de pensar y comportarse, de modo que los referidos actos expresan los hábitos, pero no lo son.

Por consiguiente, la idea de hábito comprende todos los factores selectivos y coercitivos a los que el sujeto está sometido y que, además, permiten entenderlo no como un mero dato, sino como un elemento multideterminado $\mathrm{y}$ activo, que no puede reducirse al individualismo metodológico. Por ese motivo, los neoinstitucionalistas rescatan del antiguo institucionalismo la explicación de la existencia de instituciones sociales, políticas, económicas, culturales, en un modelo de comportamiento individual que, sin embargo, no se subordina al individuo, sino que pone de relieve la interacción humana y sus resultados sociales.

Es interesante notar que los hábitos no son solo restricciones que inhiben la acción, sino que, al contrario, pueden motivar la actividad, pues crean estabilidad socioinstitucional: las instituciones restringen y habilitan el comportamiento. La existencia de reglas supone restricciones. Sin embargo, dichas restricciones pueden abrir posibilidades: permiten opciones y acciones que de otra forma no existirían (Hodgson, 2006, pág. 2). En tal sentido, el proceso coercitivo no solo se refiere al no poder hacer sino, sobre todo, al camino más estable que la acción humana puede tomar, pues esta se encuentra con un sendero sólido por el cual se puede proseguir, al mismo tiempo en que se abre un margen para explorar las adyacencias (Conceição, 2002b).

Para los neoinstitucionalistas, el movimiento individual y la dinámica institucional son recíprocamente 
causales, es decir, que los individuos moldean las instituciones de la misma forma en que estas los moldean a ellos. Este proceso se denomina causación reconstitutiva y puede asumir una dirección ascendente o descendente, en que cada estímulo producirá una reacción, la que supondrá la transformación institucional. El punto principal del argumento, de acuerdo con Hodgson (2004), es reconocer que ese proceso ocurre de forma más significativa con respecto a los hábitos que a los comportamientos, las creencias o las intenciones. De ese modo, el hábito se convierte en el eslabón crucial e invisible del canal causal (Hodgson, 2004).

En el segundo enfoque, la nueva economía institucional, se define a las instituciones como mecanismos de acción colectiva que intentan resolver el conflicto entre los individuos y aumentar la eficiencia de los negocios para promover un mayor desarrollo económico. La matriz institucional de la sociedad son las reglas del juego inventadas por los propios individuos y pueden ser formales (deliberadas políticamente, como las leyes, los códigos, las resoluciones y las normas) o informales (establecidas culturalmente, como las convenciones, la moral y la ética y los principios religiosos). Las instituciones se relacionan en gran medida con modelos mentales, pues son representaciones del individuo creadas por el sistema cognitivo para interpretar el ambiente externo, ordenándolo y estructurándolo. Las instituciones establecidas restringen el comportamiento individual y determinan el desempeño económico (North, 1994).

Villeval (1995) define la nueva economía institucional como una teoría de carácter funcional que se concentra en la solución de problemas, como la incertidumbre y los costos de transacción, y con la que se procura compensar las fallas de mercado y establecer límites a los comportamientos individuales. De ese modo, acaba por relacionarse, en general, con la coordinación de las acciones individuales típicamente interactivas. La concepción de la nueva economía institucionalista sirve para estructurar el orden social en un contexto de atribuciones e información asimétricas, con miras a la estabilización de los compromisos involucrados en derechos de propiedad y, en consecuencia, de las propias expectativas individuales acerca de la acción colectiva.

Vista su microfundamentación — basada en aspectos como los costos de transacción, el oportunismo para obtener ganancias individuales rápidas en detrimento de actividades que ofrezcan mayores beneficios sociales y el derecho de propiedad, entre otros- esta perspectiva se aproxima más al enfoque neoclásico, situándose en la corriente principal de la ciencia económica. Así, el marco analítico de la nueva economía institucionalista es una modificación de la teoría neoclásica. Conserva las hipótesis fundamentales de la escasez, la competencia y los instrumentos analíticos de la teoría microeconómica. Además, modifica el presupuesto de la racionalidad y agrega la dimensión temporal (North, 1994, pág. 359). Por ese motivo, algunos autores, como Hodgson (1998) y Dugger (1990), sugieren cautela en la incorporación de la nueva economía institucional como enfoque institucionalista "original”, debido a la proximidad con el neoclasicismo, foco de la oposición de los antiguos institucionalistas. Por otra parte, Samuels (1995) considera que la nueva economía institucional, así como el neoinstitucionalismo, realizaron importantes aportes al paradigma institucionalista, ya sea de influencia más ortodoxa o más heterodoxa (Conceição, 2002b, pág. 612). En la misma línea, Stanfield (1999) considera relevantes los avances de la nueva economía institucional, especialmente por sistematizar muchos aspectos, que la antigua tradición trató de manera informal, y superar las limitaciones de la teoría neoclásica.

Para incorporar más elementos teóricos que contribuyan al análisis de las políticas macroprudenciales en términos institucionalistas, puede ser válida la referencia a algunos aspectos de la teoría neoschumpeteriana, en la medida en que brindan elementos que ayudan a comprender el comportamiento de las instituciones financieras, objeto de control de las medidas macroprudenciales. La relación de la teoría institucionalista con la evolucionista se remonta a Veblen, que habría sido influenciado por Darwin, dado que la noción de que los instintos, los hábitos y las instituciones ejercen en la evolución económica un papel análogo al de los genes en la biología (Conceição, 2002b, pág. 609). Además, el hecho de que el institucionalismo: i) incluya dependencia de la trayectoria; ii) niegue el equilibrio; iii) se concentre en el proceso de cambio (económico, tecnológico, institucional) en una visión temporal, histórica y dinámica, y iv) entienda el carácter acumulativo de la transformación social, lo hace evolucionista. En línea con este argumento, Samuels (1995) señala que el cambio tecnológico es la principal fuente de transformación del sistema económico, que - a su vez- puede interpretarse como uno de los caminos por el que se altera el orden institucional de la sociedad.

Nelson (2002 y 2008) buscó comprender la manera en que las instituciones promueven o dificultan la implementación de cambios tecnológicos. Como es sabido, para Schumpeter (1952) el progreso tecnológico es el factor causal del desarrollo económico mientras que los aspectos extraeconómicos, como factores jurídicos, 
políticos e institucionales, limitan la capacidad innovadora de las empresas (Possas, 2006). Los movimientos institucionales y tecnológicos están interrelacionados de manera que la evolución institucional conduce a la progresión de los sistemas tanto como esta lleva a aquella, dinámica que puede explicarse por la causación reconstitutiva descendente de los neoinstitucionalistas (Hodgson, 2000a y 2000b). De acuerdo con North (1994, pág. 361), es la interacción entre instituciones y organizaciones la que moldea la evolución institucional de una economía. Si las instituciones son las reglas de juego, las organizaciones son los jugadores. En síntesis, hay una causación circular entre matriz institucional, cambio tecnológico y desarrollo económico, es decir, estos tres elementos se imbrican y condicionan recíprocamente todo el tiempo. En consecuencia, para los evolucionistas existe una fuerte interrelación entre desarrollo, crecimiento, innovación tecnológica y aparato institucional, que pone en evidencia que tales conceptos no pueden comprenderse de manera aislada. Si bien para ellos, por una parte, las instituciones no constituyen una unidad central de análisis — como en el caso de los enfoques institucionalistas-, por otra, son elementos indisociables del proceso dinámico de crecimiento y cambio tecnológico (Conceição, 2002a, pág. 139).

Teniendo en cuenta las innovaciones en el sistema financiero, Minsky (1986) destaca que la relación entre el escenario institucional en continuo cambio hacia una mayor desregulación con respecto a instituciones financieras en procura de mayores ganancias, derivó en rutinas de innovación financiera. La administración del balance de las instituciones bancarias se consolidó como un camino estratégico para que las empresas financieras consiguieran captar recursos con medios y plazos diversos y vender activos de diferente naturaleza. De los aportes de Nelson (2002 y 2008) y Possas (2006) se puede inferir que las posibilidades de innovación de las empresas del sistema financiero dependen de las trabas y los permisos establecidos por la reglamentación de las autoridades económicas. De ese modo, los límites del mercado financiero no solo se definen por los efectos que las ventajas competitivas construidas mediante las estrategias exitosas establecen en las otras competidoras y en la demanda de crédito. Las políticas públicas, monetaria o macroprudencial, tienen la capacidad de restringir o incentivar alternativas competitivas, lo que resalta el papel de la relación entre instituciones y los procesos y productos del sistema financiero.

Por ejemplo, Krugman (2009) muestra que la reciente crisis económica tuvo como coprotagonistas a las empresas del llamado sistema bancario paralelo, que realizaban actividades típicas de las instituciones bancarias, pero que, por cuenta del marco institucional de regulación, no eran consideradas como tales y, en consecuencia, estaban al margen de la reglamentación. Esas empresas desarrollaron servicios financieros apalancados y encadenados con los bancos tradicionales, lo que intensificó el contagio de la crisis. Si las instituciones no hubieran permitido operaciones de esa naturaleza, tal vez la burbuja financiera que provocó la crisis no habría alcanzado las proporciones que asumió.

El concepto de instituciones varía según las diferentes escuelas de pensamiento y, en forma análoga a Fonseca (2003), en este artículo se procura examinar los elementos que cada una de ellas puede aportar al análisis de las políticas macroprudenciales. De acuerdo con Fonseca, los institucionalismos, si bien diferentes, no llegan a ser incompatibles, pues nada impide su utilización conjunta en un estudio, sobre todo por hacer hincapié en aspectos relevantes que la amplitud y la riqueza del término institución pueden abarcar (Fonseca, 2003, pág. 135, cursiva en el original). Entonces, ante la diversidad, ¿cómo se sintetizará una noción de instituciones y cómo se articularán las perspectivas institucionalistas para alcanzar los propósitos del presente artículo?

Según los clásicos y los neoinstitucionalistas, las instituciones son, fundamentalmente, hábitos de dimensión individual y social que al interrelacionarse se traducen en propensiones comportamentales ${ }^{4}$ más o menos estables de los agentes y suponen una determinada estabilidad del perfil socioinstitucional. La referida estabilidad no significa de ninguna manera estática individual o institucional. Hay una intensa circularidad causal entre sujeto y sociedad, conforme con el modelo de las causalidades ascendente y descendente, que culminan en evolución institucional a lo largo del tiempo. Dependiendo de condiciones estructurales y coyunturales, la transformación institucional puede ocurrir de forma radical, alterando completamente el patrón anterior. Las empresas desempeñan un papel fundamental en ese proceso, pues realizan innovaciones tecnológicas que dinamizan el cambio institucional, como se puede inferir al relacionar las contribuciones institucionalistas y neoschumpeterianas.

Además, sobre la base del desarrollo teórico de la nueva economía institucionalista, cuando se refieren

\footnotetext{
4 Se entienden como propensiones comportamentales aquellas disposiciones legales que permiten a los individuos comportarse de determinada manera en situaciones determinadas.
} 
a los elementos que definen la esfera económica, la construcción y la estabilidad de los hábitos pueden reducir los costos de transacción, contribuyendo a intensificar los negocios y, en última instancia, el desarrollo económico. Los elementos relevantes para promover menores costos de transacción no se limitan a la mencionada esfera, sino que abarcan los ámbitos político, jurídico e incluso cultural. Sobre todo, las políticas públicas pueden forzosamente -y con causación descendente que Hodgson (2000b) define como de arriba abajo (top-to-bottom) - constituir hábitos y definir respuestas comportamentales. En ese sentido, al ser políticas públicas, las medidas macroprudenciales — consideradas desde un punto de vista institucionalista - pueden promover diferentes hábitos y reglas de juego en los negocios del sistema financiero, ayudando a conferirle mayor estabilidad.

En este contexto, se sugiere la siguiente articulación entre los institucionalismos:

i) Sobre la base de la nueva economía institucional, la política macroprudencial establece un conjunto de reglas de juego que, a corto plazo, regulan el comportamiento individual, tanto de las personas como de las instituciones financieras. En este ámbito, el carácter institucional de la reglamentación define los caminos que pueden explorarse en forma provechosa y los espacios a los que el regulador quiere impedir el acceso.

ii) A partir de los antiguos y los neoinstitucionalistas, según el juego de fuerzas que se entabla entre los agentes que formulan las reglamentaciones (reguladores) y los que sufren sus efectos directa o indirectamente (regulados) ${ }^{5}$, el uso de la reglamentación se refuerza y pasa a constituir, a mediano y largo plazo, una matriz institucional, es decir, un conjunto de hábitos individuales y sociales que son a su vez formales e informales.

De esta forma, no se propone una jerarquía de los enfoques institucionalistas según la pertinencia de su interpretación de la política macroprudencial, sino que, por el contrario y como se describe en el párrafo anterior, la combinación de ambos da lugar a una matriz institucional cuya construcción se realiza en forma dinámica. Esta comprende a las instituciones en el corto plazo, es decir, las reglas del juego, así como un cuadro institucional más permanente, no obstante su inherente carácter dinámico y por lo tanto evolutivo. En tal contexto, se tiene en cuenta el nexo económico-legal propuesto por Samuels (1995), en que la esfera política define parcialmente los contornos no económicos del mercado, que sin embargo suponen comportamientos económicos en las empresas y en los consumidores. A su vez, esos comportamientos no son neutros y definen parcialmente los contornos del mercado. De esta confrontación constante, y en reiterada causación acumulativa, surgen con el pasar del tiempo hábitos de comportamiento y pensamiento.

\footnotetext{
${ }^{5}$ Por ejemplo, la fijación de límites de edad para la compra de bebidas alcohólicas no es una reglamentación directa sobre la industria, sino de la libertad del consumidor. Sin embargo, al limitar el mercado consumidor, los efectos de la reglamentación afectan indirectamente a la industria. En forma análoga, al prohibirse la producción de un determinado bien o servicio, la industria es regulada directamente, mientras que el consumidor, debido a la inexistencia de la oferta, resulta afectado en forma indirecta.
}

\section{III}

\section{La política macroprudencial}

La crisis de las hipotecas de alto riesgo puso en tela de juicio la hipótesis de los mercados eficientes, que postula que el mantenimiento del nivel de precios garantiza por sí solo la asignación eficiente de los recursos y, por ende, la estabilidad financiera. Con la crisis, la política macroprudencial ha cobrado importancia y parece haber consenso (si no teórico, al menos pragmático) sobre la necesidad de adoptarla para mantener la estabilidad financiera. En palabras de Clement (2010, pág. 59), un elemento esencial de la respuesta política internacional a la crisis es fortalecer la orientación macroprudencial de la regulación y la supervisión financieras, es decir, hacer un mayor hincapié en el sistema financiero en su conjunto y su relación con la macroeconomía. En la misma línea, Galati y Moessner (2010, pág. 2) destacan que:

"No se ha apreciado la manera en que la agresiva asunción de riesgos por diferentes tipos de instituciones financieras -en el contexto de un desempeño macroeconómico sólido y bajas tasas de interés - promovió un crecimiento masivo en los balances del sistema financiero [...] Tampoco se reconoció lo suficiente el papel de la innovación 
y la desregulación financieras en el aumento de la explosión crediticia y los desequilibrios financieros y sus consecuencias en la economía real".

De acuerdo con Clement (2010), el término macroprudencial surgió a fines de 1970 en documentos inéditos, y a partir de 1980 en referencias publicadas, época en que las autoridades estaban cada vez más preocupadas por las repercusiones en la estabilidad macroeconómica y financiera del rápido ritmo de préstamos a los países en desarrollo y examinaban [nuevas] opciones políticas (Clement, 2010, pág. 60). En sus comienzos, la perspectiva macroprudencial se centraba en el ámbito microeconómico, en el que se analizan las instituciones individualmente. La ampliación de ese ámbito se debió a que las medidas microprudenciales resultaron ineficaces y condujeron a errores analíticos, ya que mientras el crecimiento individual de un banco parecía sostenible, el sistema en su conjunto podía no serlo, de tal manera que las inestabilidades que recaían en el mercado no eran evidentes a nivel microprudencial. En el cuadro 1 se presenta la diferencia entre las perspectivas macro y microprudencial.

Como puede apreciarse, a nivel macro se engloba el sistema financiero en su conjunto, mientras que el nivel micro corresponde a las instituciones, bancarias y no bancarias, individuales o clientes por separado. Esto hace que los objetivos, las preocupaciones, los riesgos y, por consiguiente, los instrumentos sean diferentes en uno y otro ámbito. Para el FMI (2011), la lógica macroprudencial es, además de una evolución, un complemento de la microprudencial y, en la práctica, resulta difícil delinear una clara separación entre los niveles prudenciales, pues independientemente de las diferencias entre ellos, la estabilidad financiera es un objetivo común, lo que refleja el largo alcance y la gravedad de las consecuencias de una crisis (FMI, 2011, pág. 10).

CUADRO 1

Comparación de las perspectivas micro y macroprudencial

\begin{tabular}{lll}
\hline Objetivo inmediato & \multicolumn{1}{c}{ Macroprudencial } & \multicolumn{1}{c}{ Microprudencial } \\
Objetivo final & $\begin{array}{l}\text { Limitar el riesgo de todo el sistema } \\
\text { financiero } \\
\text { Evitar costos macroeconómicos debidos } \\
\text { a la inestabilidad financiera } \\
\text { Earacterización del riesgo }\end{array}$ & $\begin{array}{l}\text { Limitar el riesgo de instituciones } \\
\text { individualmente } \\
\text { Proteger al depositante }\end{array}$ \\
$\begin{array}{l}\text { Correlaciones y exposiciones } \\
\text { comunes a las instituciones } \\
\text { Calibrado de controles prudenciales }\end{array}$ & Importante & $\begin{array}{l}\text { Exógeno } \\
\text { (independiente del comportamiento } \\
\text { de los agentes) }\end{array}$ \\
\hline
\end{tabular}

Fuente: Elaboración propia sobre la base de C. Borio, "Towards a macroprudentialframeword for financial supervisionandregulation?", CESifo Economics Studies, vol. 49, Oxford University Press, 2003.

\section{Objetivos de la política macroprudencial}

En general, la política macroprudencial apunta al control del riesgo sistémico y a la estabilidad financiera y, por consiguiente, a la regulación del ciclo económico que puede derivar de crisis iniciadas en el mercado financiero. Sin embargo, no hay consenso con respecto a la definición de la referida estabilidad y el perfil del riesgo. El debate se divide entre los autores que postulan el riesgo exógeno, dado por comportamientos ajenos al mercado, pero que repercuten en este, como el racionamiento del crédito (Stiglitz y Weiss, 1981) o la información asimétrica (Stiglitz y Weiss, 1992), y los que sostienen el riesgo endógeno, con arreglo a Minsky (1986) y Keynes (1943). De ese modo, es posible separar las diferentes visiones en dos grandes grupos: el primero define la estabilidad del sistema financiero en términos de su solidez ante crisis externas y el segundo hace hincapié en la naturaleza endógena de las dificultades financieras y describe la estabilidad en términos de resiliencia a crisis originadas dentro del sistema (Galati y Moessner, 2010, pág. 5). 
Según Brunnermeier y Sannikov (2009), un objetivo central de la macrorregulación es compensar la natural disminución del riesgo calculado en una fase de expansión y su subsiguiente crecimiento en una fase descendente. De acuerdo con el Banco de Inglaterra (2009), si bien la meta de la política macroprudencial debe ser el suministro estable de servicios de intermediación financiera para la economía, tratando de evitar el ciclo ascenso-descenso en la oferta de crédito y liquidez, esta no debería utilizarse para evitar burbujas y desequilibrios en general. Por otra parte, Landau (2009) argumenta que evitar burbujas económicas podría ser una de las tareas de las medidas macroprudenciales. Para Caruana (2010), el objetivo es reducir los riesgos sistémicos abordando explícitamente las interconexiones y exposiciones entre todos los agentes y el carácter procíclico del sistema financiero. Por último, Perotti y Suárez (2009) consideran que la política macroprudencial debe desalentar las estrategias bancarias individuales que causen riesgos sistémicos, o sea, una externalidad negativa sobre el sistema financiero. A pesar de cierta dispersión, en general se puede concluir que el control del riesgo sistémico y la inestabilidad del sistema financiero son el objetivo más frecuente de la política macroprudencial.

\section{Las dos dimensiones del riesgo sistémico y los instrumentos macroprudenciales}

El riesgo sistémico tiene dos dimensiones: una temporal y una de corte transversal. La primera se refiere al modo en que el riesgo evoluciona a lo largo del tiempo y la segunda a la manera en que el riesgo se distribuye en las instituciones del sistema financiero interno o externo (cuando supone transacciones con el resto del mundo, la dimensión de corte transversal puede denominarse dimensión transfronteriza) (Crockett, 2000). De acuerdo con BPI/FMI/CEF (2011, pág. 2), el tema clave en la dimensión temporal es mitigar o amortiguar el carácter procíclico del sistema financiero, mientras que en la dimensión de corte transversal se procura reducir la exposición de las instituciones financieras mediante la contención de sus vínculos con otras instituciones. En esa lógica, se necesitan instrumentos para calibrar la importancia sistémica de las instituciones individuales teniendo en cuenta las posibilidades de que propagan sus problemas al sistema. Por último, Clement (2010) subraya que, hasta la crisis, el debate sobre la política macroprudencial se concentró en el problema de la dimensión temporal, mientras que la dimensión de corte transversal cobró importancia más recientemente como resultado de las preocupaciones relativas al problema de las instituciones "demasiado grandes para quebrar".

Como en el caso de otros aspectos ya mencionados de la política macroprudencial, todavía no hay consenso en la literatura con respecto a sus instrumentos. Pese a la investigación de una serie de medidas, no se ha identificado un instrumento primario ni se ha creado una taxonomía estándar. La distinción entre las medidas macroprudenciales y otras políticas económicas no es sencilla, pues a fin de cuentas:

"Una dificultad para delinear los límites de la política macroprudencial es que otras políticas públicas también afectan la estabilidad financiera. Si bien la principal responsabilidad de garantizar la estabilidad del sistema financiero debe recaer en la política macroprudencial, otras políticas deberían poder complementarla. Independientemente de la manera en que los diferentes mandatos políticos estén estructurados, abordar la estabilidad financiera y el riesgo sistémico es una responsabilidad común" (FMI, 2011, pág. 9).

Debido a la dificultad para uniformar los instrumentos que pueden considerarse macroprudenciales, BPI/FMI/CEF (2011) crearon dos categorías posibles, aplicables a las instituciones financieras, bancarias y no bancarias: i) instrumentos específicamente concebidos para mitigar las dimensiones temporal y de corte transversal del riesgo sistémico, y ii) mecanismos desarrollados para otros fines, pero que pueden transformarse en macroprudenciales. Entre algunos ejemplos de estos últimos se incluyen las reservas obligatorias, que controlan la liquidez y pueden utilizarse para atenuar el riesgo sistémico, y el control de capitales, que otorga independencia a la tasa de interés de la política monetaria en relación con los flujos internacionales de capital en cuanto es una herramienta para la consecución de la estabilidad financiera. En el cuadro 2 se sintetizan las principales herramientas de la política macroprudencial. En general, estas consisten en mediciones, observaciones, informes, divulgaciones, reglamentaciones y límites que reducen el riesgo sistémico. Cada uno de los instrumentos incluye diversas medidas específicas de reglamentación que las instituciones financieras han de seguir. 
CUADRO 2

Instrumentos macroprudenciales

Medición de riesgos

Instituciones financieras

Medidas de riesgo calibradas a través o a lo largo del ciclo

Autoridad supervisora

Condicionalidad cíclica en la clasificación de supervisión de empresas; medidas de vulnerabilidad sistémica como base para el calibrado de instrumentos prudenciales; comunicación de vulnerabilidad sistémica; aplicación de pruebas de resistencia

\begin{tabular}{ll}
\hline & \multicolumn{1}{c}{ Informes financieros } \\
\hline Normas contables & Menor utilización de las normas contables procíclicas \\
Filtros prudenciales & $\begin{array}{l}\text { Ajuste de datos contables para calibrar los instrumentos prudenciales; normalización o moderación } \\
\text { de las medidas macroprudenciales mediante medias móviles }\end{array}$ \\
Publicidad & Divulgación de los diversos tipos de riesgo \\
\hline
\end{tabular}

Capital regulatorio

Sobretasa de capital sistémico; reducción de la sensibilidad de los requisitos de capital al punto actual del ciclo; exigencias de seguridad dependientes del ciclo económico para provisión de reservas de capital de las instituciones financieras; necesidades de capital regulatorio para tipos específicos de exposición; relación entre la revisión de la supervisión y el estado del ciclo

Fondo de liquidez

Requisitos de liquidez dependientes del ciclo; límites de concentración bancaria, prestatario, tipo de préstamo o fuente de recursos; control de préstamos y requisitos de reserva en moneda extranjera para limitar la exposición externa

Acuerdos de garantía

Plazo variable y máximo conservadurismo en relación con el valor del préstamo; metodologías de evaluación para efectos de garantía; extensión del límite de crédito sobre la base de aumentos en los valores de activos; requisito de márgenes de seguridad a lo largo del ciclo

Límites de concentración de riesgos

Límites cuantitativos en el crecimiento de los tipos específicos de exposición; incremento de la tasa de interés sobre determinados tipos de préstamos

Sistemas de compensación

Directrices que conectan el informe de desempeño de pagos con una medición ex ante del riesgo a largo plazo; ampliación del capital social con las ganancias obtenidas

Restricciones a la distribución de utilidades

Límites a los pagos de dividendos en los buenos tiempos para contribuir a la construcción de amortizadores de capital para los momentos recesivos

Mecanismos de seguro

Aportes de capital para contingencias; seguro para el riesgo sistémico medio financiado por tasas relacionadas con el crecimiento de los activos bancarios por sobre cierto límite; depósitos de seguro con premio sensible al riesgo sistémico y puntuado por parámetros microeconómicos

Gestión de fallas y resoluciones

Puntos de activación de un control más rígido en momentos de expansión

Fuente: Elaboración propia sobre la base de G. Galati y R. Moessner, "Macroprudential policy-a literature review", DNB Working Paper, No 267, Amsterdam, De Nederlandsche Bank NV, 2010.

\section{Interacción con otras políticas económicas y el diseño institucional}

Un punto importante en el diseño macroprudencial es su relación con otras políticas económicas, especialmente las monetaria, cambiaria y fiscal. Para Blanchard, Dell'Ariccia y Mauro (2010), la interacción se reforzó con el estallido de la crisis económica, pues los objetivos de la política monetaria se pusieron en tela de juicio y se asumió que esta no solo debería procurar la estabilidad de precios, sino también influir en variables reales de la economía y combatir los desequilibrios financieros. Bernanke (2011) destaca que, si bien en casos excepcionales, las medidas macroprudenciales serían complementarias a la política monetaria en ese sentido.

De acuerdo con ese punto de vista, Borio y Drehmann (2009) sugieren que la política monetaria también se ocupe de los desequilibrios financieros, pues dejar 
la inestabilidad financiera solo a cargo de la política macroprudencial sería oneroso y difícil. No obstante, la propia sistematización de la política macroprudencial interactúa con la política monetaria para la estabilidad de precios, pues con esas medidas se puede administrar el nivel de liquidez, la expansión del crédito, el flujo de capitales y el precio de los activos, que son mecanismos de transmisión de la política monetaria. De ese modo, la inflación estará bajo un mayor control durante las crisis de oferta y de demanda y, como resultado, se reducirá la sensibilidad de los intereses a sus variaciones.

Otro punto importante es elegir la manera en que se realizará la coordinación monetaria y macroprudencial, pues las dos políticas son al mismo tiempo complementarias y sustitutivas: en algunos casos se utilizan de manera conjunta para alcanzar la meta fijada, mientras que en otros es suficiente una de ellas (Galati y Moessner, 2010). Bajo esta lógica cabe señalar que un sistema financiero bien estructurado es un prerrequisito para una política monetaria eficaz, en la misma medida en que la calidad de la moneda lo es para una política macroprudencial efectiva. Un sistema financiero mal desarrollado debilita la política monetaria y puede sobrecargar a las autoridades responsables, así como una política monetaria demasiado discrecional puede provocar inestabilidad financiera y anular los esfuerzos prudenciales (Borio y Shim, 2007).

La complementariedad entre las medidas macroprudenciales y la política cambiaria es más evidente cuando estas asumen la forma de acciones normativas que controlan los flujos de capital internacional, en particular los financieros. Mediante los controles de capitales se procura reducir la exposición externa, tanto por la dimensión procíclica de los flujos como por el entrelazamiento global de activos y pasivos entre las instituciones financieras. La volatilidad inherente a los movimientos de los flujos especulativos se reduce, de tal forma que: i) el cambio se vuelve más estable y su administración más fácil; ii) aumenta la previsibilidad en el sector externo, lo que permite anticipar los riesgos de crisis cambiaria; iii) se diluyen los efectos de transmisión del cambio a los precios internos, y iv) se permite mayor flexibilidad a la administración de la política cambiaria, pues el conjunto de reglamentaciones puede modificarse de acuerdo con la coyuntura, lo que se traduce en la actuación anticíclica de la autoridad cambiaria para hacer frente al carácter procíclico de los flujos de capital.

Por último, también se puede destacar la interacción con la política fiscal. En la medida en que la política macroprudencial permite reducir la sensibilidad de los intereses básicos, tiende a aumentar la estabilidad de la estructura temporal de las tasas de interés. Esto contribuye a que la carga financiera de la deuda pública sea, como mínimo, más fácil de gestionar a lo largo del tiempo, pues ese formato de la curva de tasas de interés mejora la administración de la deuda pública, en términos de un perfil de remuneración más previsible. Se subraya, además, la coordinación entre las políticas macroprudencial, cambiaria y fiscal, en la que - mediante medidas macroprudenciales en el mercado cambiariose administran los flujos de capital y se reduce el riesgo cambiario para los países que no pueden emitir deuda externa en su propia moneda, puesto que con la disminución de la volatilidad cambiaria se reducen los costos fiscales de refinanciación, amortización e intereses.

Como se puede apreciar, los objetivos de la política macroprudencial acaban por auxiliar, directa o indirectamente, la conducción de las demás políticas económicas, ya sea aumentando su armonía, eficacia e independencia, o promoviendo la estabilidad financiera. No obstante, pese a que la complementariedad entre las políticas es evidente, el diseño institucional de la política macroprudencial es todavía controvertido. Entre los puntos del debate se destacan los siguientes: i) el problema de la autoridad macroprudencial, es decir, si dicha institución debe ser la propia autoridad monetaria u otra institución (Lastra, 2003; Blanchard, Dell' Ariccia y Mauro, 2010, y FMI, 2011); ii) la necesidad de transparencia y rendición de cuentas por parte de una institución con agentes fuera y dentro del gobierno, a fin de garantizar una aplicación eficaz e imparcial, y iii) el fomento de la cooperación internacional, con normas que eviten la arbitrariedad entre los países, garantizando una mayor estabilidad financiera mundial ${ }^{6}$.

\footnotetext{
6 Para saber más sobre el cuadro institucional de algunos países, véase FMI (2011).
} 


\section{IV \\ Política macroprudencial: una lectura institucionalista}

Al final de la segunda sección se presentó la manera en que el antiguo y el neoinstitucionalismo y la nueva economía institucional se articularon para ofrecer un marco teórico de interpretación de las medidas macroprudenciales. Sobre la base del argumento desarrollado, el primer paso para una lectura institucionalista de la política macroprudencial consiste en delinear, en breves rasgos generales, el contexto en que esta actúa. Las medidas macroprudenciales consisten, en primera instancia y con inspiración en la nueva economía institucionalista, en reglamentar el papel de las estrategias competitivas (o sea, las innovaciones financieras) de las instituciones bancarias y no bancarias para, en última instancia, constituir un sistema financiero más estable, que impulse el desarrollo económico y no sea propenso a crisis económicas y financieras. En este sentido, la autoridad pública que ha de servir como autoridad macroprudencial será el agente regulador, mientras que las empresas del sistema (bancarias y no bancarias), las familias y las empresas no financieras que hacen transacciones con activos y obligaciones de pasivos serán los regulados. Las instituciones surgen de la disputa entre esos agentes como reglas de juego. Su permanencia y consolidación, bajo la forma de una determinada matriz institucional, dependerán de la manera en que esta disputa se perpetúe, en una constante causación acumulativa.

En dicho contexto, y sobre la base de Schumpeter (1952) y Dosi (1988), las innovaciones financieras pueden entenderse como una práctica habitual de las empresas del sistema, que sirve como estrategia competitiva en la búsqueda de ganancias, crecimiento y supervivencia a lo largo del tiempo. Al ocurrir dentro de los límites del mercado financiero, inspiradas por la lógica de la búsqueda de ganancias extraordinarias, estas innovaciones resultan ser endógenas al sistema económico. Sin embargo, como muestra Zysman (1983), si bien dichas innovaciones no son esencialmente nocivas para la estabilidad financiera y, en consecuencia, para el sistema económico, pueden llegar a serlo cuando los nuevos productos y procesos financieros tienen un perfil de riesgo muy elevado y ocurren en un marco institucional poco inhibidor del riesgo sistémico.

Esto no significa que los agentes del sistema financiero, ya sean las instituciones bancarias y no bancarias o las familias y las empresas no financieras, burlen las reglas de juego, sino que se aprovechan, desvían o se sitúan al margen de ellas, estimulando la construcción de hábitos que pueden definir un contexto de mayor fragilidad. En tal sentido, la oferta de activos no ocurre independientemente de su demanda, de manera que las empresas y firmas no financieras son responsables por la validación de las innovaciones lanzadas al mercado. $\mathrm{Al}$ ofrecer ganancias a sus emisores, así como a sus titulares, los nuevos activos y obligaciones terminan por incorporarse a la cartera de los inversionistas y difundirse en el sistema mediante interconexiones nacionales y mundiales.

Isenberg (2006) y Dimsky (2006) ilustran esta lógica con respecto al sistema financiero estadounidense después de la Segunda Guerra Mundial. A partir de 1970, el sistema vigente, caracterizado por una reglamentación por segmentos en términos de formas de captación y finalidad de la concesión de préstamos, pasó por un proceso de desregulación impulsado por la búsqueda de nichos de mercado que permitieran ganancias extraordinarias a las empresas. Lejos de ser una evolución del mercado financiero, ese proceso de desregulación fue el resultado de largos debates políticos para definir las instituciones reguladoras. Isenberg cita un discurso del entonces Presidente de los Estados Unidos de América, Richard Nixon, que ilustra la manera en que el movimiento institucional tenía lugar políticamente: "Un sistema financiero fuerte, eficiente y flexible es uno que permite a las instituciones financieras adaptarse a las necesidades variables de los prestatarios y prestadores de recursos [...] y deja libertad para realizar un uso pleno de las innovaciones tecnológicas [financieras]" (Isenberg, 2006, pág. 378). Según Minsky (1986), este proceso tuvo como resultado la intensificación de las crisis financieras a nivel nacional y, como muestra Krugman (2009), también a escala mundial.

Las instituciones como reglas de juego establecen los caminos que se puedan explorar en forma provechosa, como la segmentación financiera vigente en los Estados Unidos de América hasta la década de 1970, que no condujo a ese país a ninguna crisis. Por el contrario, las reglamentaciones que instituyen la liberalización financiera, es decir, la eliminación de la regulación 
macroprudencial, resultaron promotoras de crisis cíclicas, cuyo ejemplo más reciente fue la crisis de las hipotecas de alto riesgo. Así, las instituciones que no establecen límites al movimiento especulativo suponen la conformación de un sistema que permite el apalancamiento procíclico de activos de riesgo, a corto plazo, que pueden ser rápidamente negociables y permiten series de derivados financieros a nivel nacional y mundial. De acuerdo con una lectura institucionalista, puede afirmarse que, cuando están circunscritos a matrices institucionales poco restrictivas, los hábitos de los agentes del mercado financiero aumentan la propensión del sistema a los desequilibrios, ilustrando el casino en el que según Keynes (1943) se había convertido el mercado financiero.

La referencia a Keynes (1943) llama la atención hacia una importante institución informal presente en los sistemas financieros: el comportamiento especulativo. Como señala Keynes (1943), la racionalidad de estos mercados se dirige a la actuación especulativa que, para el autor, significa que los inversionistas no observan los fundamentos de las empresas cotizadas en los mercados de valores, sino que se guían por el intento de prever con mayor rapidez cuál será la evaluación convencional media de los participantes del mercado. Para limitar ese hábito informalmente establecido en los mercados financieros, Keynes (1943) sugería el uso de elementos que impidieran el libre movimiento de los agentes (como la tributación), en franca demostración de la manera en que las instituciones formales pueden restringir a las informales. El carácter recurrente de las crisis en los países desarrollados y emergentes muestra, sin embargo, que poco se ha hecho para constituir una matriz institucional de largo plazo que reduzca los hábitos especulativos en el mercado financiero. Por el contrario, como señala Arestis (2006), la desregulación o liberalización de las finanzas ha aumentado a nivel mundial.

Todos los enfoques institucionalistas señalan que el mercado, en este caso financiero, no es autosuficiente ni autorregulado. De hecho, está determinado por la estructura institucional de las sociedades, de modo que solo cumple con las instituciones predominantes (Conceição, 2002a, pág. 126), volviéndose lo que es y desempeñando sus funciones específicas por causa de las instituciones que operan como control social y se forman y funcionan a través de él (Samuels, 1995). Las políticas macroprudenciales pueden, por lo tanto, atribuir dos tipos de institucionalidad a los mercados financieros. A nivel agregado, condicionarán los contornos del sistema financiero, volviéndolo más o menos accesible a la especulación. A nivel particular, por tratarse de reglas de juego, construirán hábitos individuales, ya sea de los acreedores o los deudores, y crearán así un cuadro de incentivos y restricciones (Zysman, 1994). Se destaca entonces la relación entre los ámbitos micro y macro, entre el individuo y el todo, que representa la pieza clave del paradigma institucionalista, como señala Hodgson (1998 y 2004). Plott (1991) sigue la misma línea al indicar que las preferencias individuales y las instituciones conforman los resultados económicos.

Al respecto, cabe señalar que la política macroprudencial limita las actividades financieras especulativas y, al ser deliberada políticamente y después de un tiempo legitimada (captada por las preferencias individuales y los hábitos comportamentales colectivos dado que su finalidad última es el bien común), se integra o arraiga como matriz institucional. Entonces, los agentes pasan a incorporar habitualmente su presencia en la toma de decisiones. En palabras de North (1994, pág. 359): "Las instituciones forman la estructura de incentivos de la sociedad; en consecuencia, las instituciones económicas y políticas son los determinantes subyacentes del desempeño económico"7.

Asimismo, puede ocurrir que se establezcan y perduren instituciones que permiten innovaciones financieras que aumentan las posibilidades de crisis. Esa matriz institucional, como la que en la crisis actual permitió la existencia del sistema bancario paralelo (Krugman, 2009), puede considerarse como un caso de "instituciones imbéciles". En ese sentido, se puede incurrir en el "efecto cerrojo", de acuerdo con Arthur (1989), ya que son cambios técnicos que cuesta revertir y que hacen necesaria la creación de instituciones o la adaptación de las ya existentes, visto que el progreso financiero no tuvo lugar de forma socialmente satisfactoria.

Es posible que la imposición de medidas macroprudenciales cause efectos negativos a corto plazo, tanto por su resonancia en las preferencias y los comportamientos individuales, como porque el sector financiero puede tomar represalias contra las autoridades económicas o demorar en adecuarse a las nuevas reglas de conducción de operaciones y estrategias competitivas. Sin embargo, la idea es que las nuevas reglas de juego a corto plazo confieran mayor estabilidad al sistema financiero a mediano y largo plazo. En este contexto, son relevantes las variables tiempo y acumulación de la evolución institucional. Por una parte, es a lo largo del tiempo que ocurren los cambios socioeconómicos

\footnotetext{
${ }^{7}$ De ahí la importancia del Estado y de sus políticas públicas, que ocupan una posición social privilegiada por poseer un legítimo poder para legislar y garantizar las normas.
} 
y es en este que tiene lugar el proceso de aprendizaje. Mientras tanto, la relación entre las reglas de juego (la política macroprudencial) y los jugadores (las instituciones financieras) configura el perfil de lo que será la estructura institucional. Por otra parte, de acuerdo con Veblen, la situación de hoy moldea el mañana por medio de un proceso coercitivo y selectivo, que actúa sobre los hábitos y puede alterar el punto de vista o la actitud mental que se hereda del pasado. Eso significa que en medio de su evolución, las instituciones son acumulativas. En tal sentido, si bien la restricción macroprudencial puede realizarse con la estructura de reglamentación vigente, eso no siempre es posible dada la acumulación, que tal vez exigirá considerables modificaciones a la matriz de reglas a fin de establecer nuevos caminos para el desarrollo del sistema financiero.

Por regla general, no es posible conocer las innovaciones financieras y sus resultados a priori, debido a la imposibilidad de trazar con precisión las consecuencias de las acciones (Dosi, 1988, pág. 222), algo que se aplica a todas las políticas. Por ese motivo y por el comportamiento estratégico de las instituciones financieras, las medidas macroprudenciales, sujetas a la causación acumulativa, deben adaptarse en forma constante para acompañar el carácter evolutivo del sistema económico. "Las autoridades económicas se deben preocupar por el efecto en la estabilidad de la estructura cambiante de las relaciones financieras [...] guiando la evolución de las instituciones financieras, favoreciendo las instituciones y prácticas que aumentan la estabilidad y desalentando aquellas que contribuyen al aumento de la inestabilidad" (Minsky, 1986, pág. 314).

Es interesante constatar que la política macroprudencial actúa sobre los niveles individual y

\section{IV}

\section{Conclusiones}

Abordar la política económica macroprudencial a partir del marco teórico institucionalista no solo es una tarea importante, sino también necesaria dadas las exigencias impuestas por los recientes episodios de inestabilidad económica, entre los que se destaca la crisis de 2008. En ese sentido, tanto Hodgson (2009, pág. 3) como social, puesto que regula la demanda de riesgo de los agentes y la oferta de activos de la misma naturaleza de las instituciones financieras, cuyo encuentro supone mayor probabilidad de que se incurra en riesgos sistémicos. Como se señaló en las lógicas micro y macro de la política prudencial, no hay una disociación entre la parte y el todo. La crisis de 2008 puso en evidencia que una institución demasiado grande puede quebrar y arrastrar consigo a toda una serie de instituciones con las que hizo negocios. No obstante, a la luz de Keynes (1943), el efecto de contagio y la crisis de desconfianza se traducen en un menor ritmo de actividad económica. Así, la quiebra de una o pocas empresas puede contaminar todo el sistema económico y, como se ha visto, la política macroprudencial interviene en la interacción entre la parte y el todo.

Por último, los recientes debates sobre la política macroprudencial son compatibles con los enfoques institucionales en sus diversas perspectivas y definiciones del concepto de instituciones - reglas de juego que, una vez adoptadas socialmente, se convierten en hábitos- La literatura especializada en políticas macroprudenciales no sugiere la relación entre los instrumentos y la perspectiva institucionalista. Sin embargo, dicha aproximación teórica es pertinente, pues no hay ninguna divergencia lógica o teórica, lo que permite dar solidez a los argumentos en favor de las medidas macroprudenciales como política pública de naturaleza económica, cuyo objetivo es la estabilidad de las economías capitalistas. Si el objetivo de las políticas es reducir las inestabilidades inherentes al sistema económico, es preciso que las instituciones cambien y para ello las políticas macroprudenciales son herramientas capaces de dar una institucionalidad idónea a los mercados.

Borio (2010, pág.1), parafraseando a Friedman ${ }^{8}$, afirman respectivamente "ahora somos todos institucionalistas" y "ahora somos todos macroprudencialistas". Las políticas

${ }^{8}$ En su frase "ahora somos todos keynesianos" (Borio, 2010). 
macroprudenciales son todavía un tema controvertido y, sobre la base de la revisión de la literatura realizada en este trabajo, se diagnostica la falta de una base teórica que las sustente. Resta así una aparente perspectiva de neutralidad que las deja bajo un análisis pragmático. No obstante, se puede concluir que la política macroprudencial es coherente con el pensamiento institucionalista en sus diversas corrientes y es posible interpretarla como reglamentaciones de corto plazo que limitan y al mismo tiempo estimulan hábitos, conformando una matriz institucional. Esta comprende tanto instituciones formales, de las que las macroprudenciales forman parte, como informales. En particular, las medidas macroprudenciales son políticas públicas de naturaleza económica con un sesgo regulatorio normativo destinadas a promover la estabilidad del sistema financiero y, por ese motivo, pueden ser un importante instrumento para restringir caminos potencialmente inestables y estimular otros más favorables al financiamiento con estabilidad.

Al tratarse de un estudio teórico de política económica, este trabajo tiene una naturaleza bastante normativa, a la que se procuró dar materialidad mediante ejemplos históricos y recientes. Esto no significa, sin embargo, que las políticas macroprudenciales sean la solución final para los problemas financieros que aquejan a las economías capitalistas modernas, marcadas por flujos financieros intensos y globalizados. De acuerdo con ello, si bien parecen ser instrumentos eficientes de política económica y están desde hace tiempo presentes en la literatura, cabe todavía enfrentar muchos desafíos con respecto a las macroprudenciales. Entre ellos figuran: i) la definición más precisa de sus objetivos; ii) la elaboración de sus instrumentos (en especial los relacionados con la medición y observación de riesgos sistémicos) y la comprensión de sus canales de transmisión; iii) la elaboración de un cuadro institucional y de mecanismos apropiados de gobernanza que permitan el uso constante y efectivo de sus medidas, sin que la autoridad responsable sufra represalias por parte del sistema financiero, y, por último, iv) el fomento de una cooperación internacional que garantice la eficacia de las herramientas macroprudenciales en un mundo globalizado.

La política macroprudencial es un posible camino para limitar la fragilidad financiera y el riesgo endógeno a partir de un cambio institucional que puede conducir a una transformación de la estructura institucional del sistema financiero. En consecuencia, la preferencia por la liquidez de las instituciones bancarias no responderá únicamente a sus análisis procíclicos, sino a una institución reguladora. Sin duda, este puede ser un camino viable para limitar los riesgos sistémicos planteados por las estrategias competitivas y las innovaciones financieras -que resultan en posiciones apalancadas y financieramente frágiles de los agentes-, sobre todo si, como en el paradigma institucional, se les reconoce a las preferencias individuales, las instituciones (en sentido amplio) y los cambios tecnológicos el papel de motor principal del desempeño de la economía.

\section{Bibliografía}

Arestis, P. (2006), "Financial liberalization and the relationship between finance and growth", A Handbook of Alternative Monetary Economics, P. Arestis y M. Sawyer, Cheltenham, Edward Elgar.

Arthur, W.B. (1989), "Competing technologies, increasing returns and lock-in by historical events", Economic Journal, vol. 99, $\mathrm{N}^{\circ} 394$, Wiley, marzo.

Banco de Inglaterra (2009), "The role of macroprudential policy", Discussion Paper, Londres, noviembre.

Bernanke, B.S. (2011), "Implementing a macroprudential approach to supervision and regulation", discurso pronunciado en la $47^{\mathrm{a}}$ Conferencia Anual sobre Estructura Bancaria y Competencia [en línea] http://www.federalreserve.gov/newsevents/speech/ bernanke20110505a.htm.

Blanchard, O., G. Dell'Ariccia y P. Mauro (2010), "Rethinking macroeconomic policy", IMF Staff Position Note (SPN/10/03), Washington, D.C., Fondo Monetario Internacional.

Borio, C. (2010), "Implementing a macroprudential framework: blending boldness and realism", Working Papers, vol. 49, $\mathrm{N}^{\circ} 2$, Basilea, Banco de Pagos Internacionales.
(2003), "Towards a macroprudential frameword for financial supervision and regulation?", CESifo Economics Studies, vol. 49, Oxford University Press.

Borio, C. y M. Drehmann (2009), "Towards an operational framework for financial stability: 'fuzzy' measurement and its consequences", Working Papers, $\mathrm{N}^{\circ}$ 284, Basilea, Banco de Pagos Internacionales.

Borio, C. e I. Shim (2007), "What can (macro)-prudential policy do to support monetary policy?", Working Papers, $\mathrm{N}^{\circ} 242$, Basilea, Banco de Pagos Internacionales.

BPI/FMI/CEF (Banco de Pagos Internacionales/Fondo Monetario Internacional/Consejo de Estabilidad Financiera) (2011), "Macroprudencial Policy Tools and Frameworks. Update to G20 Finance Ministers and Central Bank Governors" [en línea] https://www.imf.org/external/np/g20/pdf/021411.pdf.

Brunnermeier, M. y Y. Sannikov (2009), "A Macroeconomic Model with a Financial Sector", Princeton, Universidad de Princeton, versión preliminar.

Caruana, J. (2010), "Macroprudential Policy: Working Towards a New Consensus", Banco de Pagos Internacionales [en línea] http://www.bis.org/speeches/sp100426.pdf. 
Clement, P. (2010), "The term 'macroprudential': origins and evolution", BIS Quarterly Review, Basilea, Banco de Pagos Internacionales, marzo.

Commons, J.R. (1931), "Institutional economics", American Economic Review, vol. 21, Nashville, Tennessee, American Economic Association.

Conceição, O.A.C. (2008), "Além da transação: uma comparação do pensamento dos institucionalistas com os evolucionários e pós-keynesianos", Economia, vol. 8, №3, Asociación Nacional de Centros de Posgrado en Economía (ANPEC).

(2002a), "Conceito de instituição nas modernas abordagens institucionalistas", Revista de Economia Contemporânea, vol. $6, \mathrm{~N}^{\circ} 2$.

(2002b), "A relação entre o processo de crescimento econômico, mudança e instituições na abordagem institucionalista", Ensaios FEE, vol. 23, número especial, Fundación de Economía y Estadística.

Conceição, O.A.C. y F. Ferrari Filho (2001), A noção de incerteza nos pós-keynesianos e institucionalistas: uma conciliação possível?, Nova Economia, vol. 11, $\mathrm{N}^{\circ} 1$.

Crockett, A. (2000), "Marryingthe micro- and macroprudentialdimensions of financialstability", Banco de Pagos Internacionales [en línea] http://www.bis.org/speeches/ sp000921.htm.

Dillard, D. (1980), "A monetary theory of production: Keynes and the institutionalists", Journal of Economic Issues, vol. 14, № 2 , Association for Evolutionary Economics.

Dimsky, G. (2006), "Banking and financial crises", A Handbook of Alternative Monetary Economics, P. Arestis y M. Sawyer, Cheltenham, Edward Elgar.

Dosi, G. (1988), "The nature of the innovative process", Technical Change and Economic Theory, G. Dosi y otros (eds.), Londres, Pinter Publishers.

Dugger, W. (1990), "The new institutionalism: new but not institutionalism", Journal of Economic Issues, vol. 24, $\mathrm{N}^{\circ} 2$, Association for Evolutionary Economics.

FMI (Fondo Monetario Internacional) (2011), "Macroprudential Policy: An Organizing Framework", Washington, D.C. [en línea] https://www.imf.org/external/np/pp/eng/2011/031411. pdf.

Fonseca, P.C.D. (2003), "Sobre a intencionalidade da política industrializante no Brasil na década de 1930", Revista de Economia Política, São Paulo, $\mathrm{N}^{\circ} 89$.

Galati, G. y R. Moessner (2010), "Macroprudential policy-a literature review", DNB Working Paper, No 267, Amsterdam, De Nederlandsche Bank NV.

Goudard, G.C. y F. Terra (2013), "Medidas macroprudenciais: um debate essencialmente keynesiano", Anais do VI Encontro Internacional da Associação Keynesiana Brasileira, Vitória.

Hodgson, G.M. (2009), "Institutional economics into twenty-first century", Studi e Note di Economia, vol. 14, $\mathrm{N}^{\circ} 1$.

(2006), "What are institutions?", Journal of Economic Issues, vol. 40, $\mathrm{N}^{\circ} 1$, Association for Evolutionary Economics. (2004), "Reclaiming habit for institutional economics", Journal of Economic Psychology, vol. 25, № 5, Amsterdam, Elsevier.

(2002), "The evolution of institutions: an agenda for future theoretical research", Constitutional Political Economy, vol. 13, $\mathrm{N}^{\circ} 2$, Springer.

(2000a), "The hidden persuaders: institutions and choice in economic theory", Anais do seminário "A situação atual da microeconomia: uma perspective metodológica", Curitiba. (2000b), "What is the essence of institutional economics?", Journal of Economic Issues, vol. 34, $\mathrm{N}^{\circ} 2$, Association for Evolutionary Economics.

(1998), "The approach of institutional economics", Journal of Economic Literature, vol. 36, $\mathrm{N}^{\circ}$ 1, Nashville, Tennessee, American Economic Association.
(1993), "Institutional economics: surveying the 'old' and the "new", Metroeconomica, vol. 44, $\mathrm{N}^{\circ} 1$, Wiley.

Isenberg, D. (2006), "Deregulation", A Handbook of Alternative Monetary Economics, P. Arestis y M. Sawyer, Cheltenham, Edward Elgar.

Keynes, J.M. (1943), Teoría general de la ocupación, el interés y el dinero, Ciudad de México, Fondo de Cultura Económica.

Krugman, P. (2009), A crise de 2008 e a economia da depressão, Río de Janeiro, Elsevier.

Landau, J.P. (2009), "Bubbles and Macroprudential Supervisor" [en línea] http://www.bis.org/review/r090327e.pdf.

Lastra, R. (2003), "The governance structure for financial regulation and supervision in Europe", Columbia Journal of European Law, vol. 10 [en línea] http://www.law.harvard.edu/faculty/ hjackson/alumnipapers/Governance_in_EU.Lastra.pdf.

Minsky, H. (1986), Stabilizing an Unstable Economy, Nueva York, Yale University Press.

Nelson, R. (2008), "What enables rapid economic progress: what are the needed institutions?", Research Policy, vol. $37, \mathrm{~N}^{\circ} 1$, Amsterdam, Elsevier.

(2002), "Bringing institutions into evolutionary growth theory", Journal of Evolutionary Economics, vol. 12, $\mathrm{N}^{\circ} 1$, Springer.

North, D.C. (1994), "Economic performance through time", American Economic Review, vol. 84, $\mathrm{N}^{\circ} 3$, Nashville, Tennessee, American Economic Association.

(1990), Institutions, Institutional Changes and Economic Performance, Nueva York, Cambridge University Press.

Perotti, E. y J. Suárez (2009), "Liquidity risk charges as a macroprudential tool”, Universidad de Amsterdam, inédito.

Plott, C.R. (1991), "Will economics become an experimental science?", Southern Economic Journal, vol. 57, $\mathrm{N}^{\circ} 4$, Chattanooga, Tennessee, Southern Economic Association.

Polanyi, K. (1944), The Great Transformation: The Political and Economic Origins of Our Time, Boston, Beacon Press.

Possas, S. (2006), "Concorrência e inovação", Economia da inovação tecnológica, V. Pelaez y T. Szmrecsáyi (orgs.), São Paulo, Hucitec.

Samuels, W.J. (2007), The Legal-Economic Nexus: Fundamental Processes, Nueva York, Taylor \& Francis.

(1995), "The present state of institutional economics", Cambridge Journal of Economics, vol. 19, $\mathrm{N}^{\circ} 4$, Oxford University Press.

Schumpeter, J.A. (1952), Capitalismo, socialismo y democracia, Madrid, Aguilar.

Stanfield, J.R. (1999), "The scope, method and significance of original institutional economics", Journal of Economic Issues, vol. 33, $\mathrm{N}^{\circ} 2$, Association for Evolutionary Economics.

Stiglitz, J. y M. Weiss (1992), "Asymmetric information in credit markets and its implications for macroeconomics", Oxford Economic Papers, vol. 44, $\mathrm{N}^{\circ}$ 4, Oxford, Oxford University Press.

(1981), "Credit rationing with markets with imperfect information", American Economic Review, vol. 71, $\mathrm{N}^{\circ} 3$, Nashville, Tennessee, American Economic Association.

Veblen, T. (1919), The Place of Science in Modern Civilization and Other Essays, Nueva York, B.W. Huebsch.

(1899), The Theory of the Leisure Class: An Economic Study in the Evolution of Institutions, Nueva York, Macmillan.

Villeval, M.C. (1995), "Une théorie économique des instituitions", Theórie de la régulation: l'état des savoirs, $\mathrm{R}$. Boyer y Y. Saillard, París, La Découverte.

Zysman, J. (1994), "How institutions create historically rooted trajectories of growth", Industrial and Corporate Change, vol. 3, $\mathrm{N}^{\circ} 1$, Oxford University Press.

(1983), Government, Markets and Growth: Financial Systems and the Politics of Industrial Change, Ithaca, Cornell University Press. 